\title{
Association of Interleukin-10 Polymorphism (rs1800896, rs1800871, and rs1800872) with Breast Cancer Risk: an Updated Meta-Analysis Based on Different Ethnic Groups
}

\section{Li-Jun Li}

The Second Affiliated Hospital of the University of South China

\section{Memg-Ling Cao}

The Second Affiliated Hospital of the University of South China

\section{Dong-Hua Li}

The Second Affiliated Hospital of the University of South China

Wei Xiong

The Second Affiliated Hospital of the University of South China

Jiangang Cao (D814709270@qq.com)

University of South China Affiliated Nanhua Hospital https://orcid.org/0000-0002-3376-108X

\section{Research Article}

Keywords: Interleukin -10, Gene polymorphism, Breast cancer, Meta-analysis

Posted Date: June 10th, 2021

DOI: https://doi.org/10.21203/rs.3.rs-587891/v1

License: (c) (i) This work is licensed under a Creative Commons Attribution 4.0 International License. Read Full License

Version of Record: A version of this preprint was published at Frontiers in Genetics on February 4th, 2022. See the published version at https://doi.org/10.3389/fgene.2022.829283. 


\section{Abstract}

Background: There have been various publications stating that IL-10 gene polymorphism is connected with the risk of breast cancer.This study was to further evaluate the association between IL-10 gene polymorphism and breast cancer (BC).

Methods: Articles from PubMed, Web of Knowledge, Embase, CNKI databases, China biomedical (CBM), and Google Scholar before March 29, 2020. The data was analyzed by Revman5.3 and Stata 12.0 software, and the strength of the association was identified using the odds ratio (OR) and the corresponding 95\% confidence interval (Cl).

Results: A total of 23 studies (7250 cancer cases and 7675 case-free controls) were eligible for the mate-analysis. The results show that IL-10 gene polymorphisms has no association with BC risk in any genetic model of the general population. However, there is significantly correlated with $\mathrm{BC}$ risk based on subgroup analysis by ethnicity: rs 1800896 polymorphism are significantly associated with the risk of $\mathrm{BC}$ in Asian population based on the four models ( $\mathrm{G}$ vs. $\mathrm{A}$ : $\mathrm{OR}=0.78,95 \% \mathrm{Cl}=0.65-0.95, P=0.01 ; \mathrm{GG}$ vs. AA: $O R=0.51,95 \% \mathrm{Cl}=0.31-0.84, P=0.007$; $\mathrm{GA}$ vs. AA: $\mathrm{OR}=0.6,95 \% \mathrm{Cl}=0.44-0.81, P=0.0009$; $\mathrm{GG}+\mathrm{GA}$ vs. $\mathrm{AA}: \mathrm{OR}=0.6,95 \% \mathrm{Cl}=0.45-$ $0.81, P=0.0007)$; rs1800871 polymorphism has association with $\mathrm{BC}$ risk in Caucasian population based on the Heterozygous model (CT vs. TT: OR=1.8, 95\% $\mathrm{Cl}=1.03-3.13, P=0.04$ ). rs1800872 homozygous model was associated with the $\mathrm{BC}$ risk in Asians(AA vs CC: $\mathrm{OR}=0.74,95 \% \mathrm{Cl}=0.55-0.99, P=0.04$ ), and allelic model was associated with $\mathrm{BC}$ risk among Asians (A vs $\mathrm{C}$ : OR= $0.85,95 \% \mathrm{Cl}=0.74-0.98, P=0.03$ ) and among Caucasians (A vs C: $\mathrm{OR}=0.65,95 \% \mathrm{Cl} 0.43-0.98, P=0.04$ ).

Conclusion: IL-10 gene polymorphism rs1800896 and rs1800872 significantly increased the risk of breast cancer in Asians, while rs1800871 was associated with the risk of breast cancer in Caucasians. Therefore, vulnerable individuals can be identified by genotypic characteristics, and the diseases can be prevented through interventions aimed at high-risk groups.

\section{Background}

Breast cancer (BC) is the leading cause of death among women worldwide and accounts for $11.9 \%$ of cancers in the world[1]. BC incidence varies widely, ranging from 27/100,0002 (Central-East Asia and Africa) to 85-94/100,0002 (Australia, North America and Western Europe). Its frequency in France is among the highest in Europe[2]. In Asian countries, the incidence of $B C$ is also rising rapidly $[3,4]$. Although genetic, environmental, and lifestyle are associated with $B C$ occurrence, the biological mechanism that causes $B C$ remains unclear.

Inflammation plays a significant role in BC development and is an important part of the BC microenvironment[5]. Interleukin-10 (IL-10) is an important anti-inflammatory and immunomodulatory cytokine found in the human immune response. IL-10 is located on chromosome 1 (1q31-1q32) and is composed of 5 exons and 4 introns[6]. Single nucleotide polymorphism (SNP) is the most common form of genetic variation. In the SNP database (http://www.ncbi.nlm.nih.gov/snp), the three promoter SNPs of IL-10, rs1800896 (-1082A/G), rs1800871 (-819T/C) and rs1800872 (-592A/C) is considered to be related to differences in gene transcription levels and is the most widely studied in many diseases [7]. It has been reported that IL-10 gene polymorphism plays an important role in the occurrence and development of cancers such as BC, gastric cancer, colorectal cancer, lung cancer, cervical cancer, and digestive cancer[8-10].

A study in Egypt found that: rs1800896 (-1082A/G) polymorphism is correlated with cancer staging and has association with progression of breast cancer at AA genotype [11]. In the research based on Caucasians, it was found that there was a significant association between IL10-1082 G/G genotype and the increased risk of BC.[12]. Another study found that the rs1800871 $(-819 T / C)$ polymorphism increases the risk of BC in Han Chinese women[13]. A rare allele of rs1800872 (-592A/C) polymorphism may be a potential prognostic indicator of disease-free survival in BC patients[14]. Therefore, the IL-10 gene polymorphism can influence the risk of human $\mathrm{BC}[15]$, but these are based on research in different countries and populations. Studies have shown that the relationship between IL-10 gene polymorphism and BC risk is strongly associated with ethnicity[16]. Therefore, in this study, a meta-analysis was used to investigate the relationship between IL-10 rs1800896, rs1800871, and rs1800872 polymorphism and BC risk and aimed to explore the association between IL-10 gene polymorphism and BC risk in different populations in the world. 


\section{Methods}

This meta-analysis was conducted according to the PRISMA reporting criteria [17].

\section{Search Strategy}

Research articles on the relationship between IL-10 gene polymorphism and BC risk in different databases including PubMed, Web of Knowledge, Embase, CNKI, CBM, VIP, WanFang Data, and Google academic were retrieved with the keywords: Interleukin10/IL-10, 1082A/G/rs1800896, 819T/C/rs1800871, 592A/C/rs1800872, Breast carcinoma/Breast cancer" etc. All eligible studies were searched until March 29, 2020. Take the PubMed database as an example to list the retrieval strategies, as shown in Fig. 1.

\section{Inclusion and exclusion criteria}

\section{Inclusion criteria}

(1) the case group was clinically diagnosed with BC and the control group was healthy; (2) Case-control studies or cohort studies to evaluate the relationship between IL-10 polymorphism and BC risk; (3) full-text published in English and(or) Chinese; (4) Report the number of cases and controls for each genotype and detailed genotyping data, or knowing the odds ratio (OR) helps to calculate the $95 \%$ confidence interval $(\mathrm{Cl})$.

\section{Exclusion criteria}

(1) Abstracts, reviews, animal studies; (2) Studies of unreported genotypes frequency; (3) Studies on the relationship between IL10 gene polymorphism and prognosis and other aspects of breast cancer; (4) studies on the obvious imbalance of baseline between the case group and control group; (5) The case and control sources not provided; (6) Repeatedly published literature; and if multiple studies from the same case series are available, the study with the largest number of individuals is used in the analysis.

\section{Data extraction}

Two researchers selected the literature according to the inclusion and exclusion criteria, extracted the data, and cross-checked them into a standard data collection form independently. If there are any disputes, we will reach an agreement by discussion or by a third party, and strive to reach a consensus on each project. Data were collected from each article included: the first author, year of publication, study location, type of study, ethnicity (classified as Asian, Caucasian,or mixed descent), total number of cases and controls, genotype frequency, genotype detection method, and the source of control.

\section{Sensitivity analysis}

Sensitivity analysis was performed to assess the stability of the results. The Funnel plot, Begg'test, and Egger'test were used to evaluate publication bias. RevMan5.3 and Stata 12.0 software were used for the above statistical analysis[18].

\section{Statistical analysis}

The correlation between IL-10 gene polymorphism and BC risk was evaluated by OR and $95 \% \mathrm{Cl}$ as the effect size. $95 \% \mathrm{Cl}$ without 1 and $P<0.05$ were considered statistically significant. The Z-test determines the significance of the OR value. The effects of heterogeneity were quantified by $\mathrm{I}^{2}$ and $P$ values. In addition, the $\mathrm{I}^{2}$ value is used to quantify the degree of heterogeneity, where $\mathrm{I}^{2}$ $<25 \%$ (low/no heterogeneity), $25-75 \%$ (moderate heterogeneity), and $>75 \%$ (large or very heterogeneous). When the $\mathrm{I}^{2}<25 \%$, the fixed-effect model is adopted; otherwise, the random effect model is adopted.

\section{Results}

\section{Search results}


We had a total of 78 articles after removing 3 duplicated articles. After layer-by-layer screening, a total of 23 articles finally met the criteria for inclusion in this meta-analysis. Eligible papers were published between 2003 and 2019. The Mata-analysis updates three 2019 case-control studies compared to previous meta-analyses [11, 16, 17].

\section{Data extraction and quality assessment}

The 23 eligible articles had a total sample size of 14,925 participants, including 7,250 BC patients and 7,675 healthy controls[11, 16-37]. The samples were involved in three IL-10 polymorphism sites: rs 1800896 , rs 1800871 , and rs 1800872 . There were 17 studies on rs 1800896 (3,308 cases and 3,425 controls), 12 studies on rs 1800871 (2,530 cases and 2,698 controls), and 13 studies on rs 1800872 (4,702 cases and 4,818 controls). The 10 were based on Caucasians and 6 were based on Asians, while the remaining 7 were mixed-race in the 23 criteria studies. Of these studies, the 18 were hospital-based and 5 were population-based. The Newcastle-Ottawa Scale (NOS) was used to assess the quality of the included articles[38]. NOS scores ranged from 0 to 9. We consider the methodological quality of the study to be good if the study score is $\geq 7$. Our data extraction and quality evaluation were independently completed by two authors. Table 1 and Table 2 show the basic characteristics of the included literature, the distribution of polymorphisms at the studied gene sites, allele frequency, and the quality assessment of the included literature. 
Table 1

Characteristics of the Studies Included in the Meta-Analysis

\begin{tabular}{|c|c|c|c|c|c|c|c|c|c|}
\hline First author & Year & Country & Ethnicity & $\begin{array}{l}\text { Genotyping } \\
\text { method }\end{array}$ & SOC & case/control & $\begin{array}{l}\text { Study } \\
\text { design }\end{array}$ & $\begin{array}{l}\text { SNP } \\
\text { No. }\end{array}$ & $\begin{array}{l}\text { NOS } \\
\text { score }\end{array}$ \\
\hline $\begin{array}{l}\text { Gallegos- } \\
\text { Arreola }\end{array}$ & 2019 & Mexican & Mixed & PCR-RFLP & $\mathrm{HB}$ & $368 / 320$ & CC & 3 & 8 \\
\hline Al-Ankoshy & 2019 & Iraq & Caucasian & PCR-SSP & $\mathrm{HB}$ & 70/70 & CC & 1 & 8 \\
\hline Zeng & 2019 & China & Asian & PCR-RFLP & $\mathrm{HB}$ & $208 / 215$ & CC & 3 & 8 \\
\hline Sabet & 2017 & Egypt & Caucasian & PCR-RFLP & $\mathrm{HB}$ & $105 / 50$ & $\mathrm{CC}$ & $1,2,3$ & 7 \\
\hline Tian & 2017 & China & Asian & Mass ARRAY & PB & $312 / 312$ & CC & $1,2,3$ & 7 \\
\hline Maruthi & 2017 & India & Mixed & PCR-RFLP & $\mathrm{HB}$ & $285 / 285$ & CC & 1 & 7 \\
\hline Atoum & 2016 & Jordan & Mixed & PCR-RFLP & $\mathrm{HB}$ & $202 / 210$ & CC & $1,2,3$ & 7 \\
\hline Alsuhaibani & 2016 & Egypt & Caucasian & PCR-RFLP & $\mathrm{HB}$ & $80 / 80$ & CC & 1 & 7 \\
\hline Vinod & 2015 & India & Mixed & ASPCR & $\mathrm{HB}$ & $125 / 160$ & CC & 1 & 8 \\
\hline Li & 2014 & China & Asian & PCR-SSP & PB & $128 / 128$ & CC & 1,2 & 7 \\
\hline Wang & 2014 & China & Asian & PCR-RFLP & $\mathrm{HB}$ & $474 / 501$ & CC & 2 & 8 \\
\hline Pooja & 2012 & India & Mixed & PCR-RFLP & PB & $200 / 200$ & CC & $1,2,3$ & 7 \\
\hline $\mathrm{He}$ & 2012 & China & Asian & MALDI-TOF MS & $\mathrm{HB}$ & $347 / 500$ & $\mathrm{CC}$ & 2 & 7 \\
\hline Kong2010 & 2010 & China & Asian & PCR-RFLP & $\mathrm{HB}$ & $315 / 322$ & CC & $1,2,3$ & 7 \\
\hline Pharoah & 2007 & European & Caucasian & TaqMan & PB & $2045 / 2218$ & CC & 3 & 8 \\
\hline Gonullu & 2007 & Turkey & Caucasian & Mass ARRAY & $\mathrm{HB}$ & $38 / 24$ & CC & $1,2,3$ & 7 \\
\hline Scola & 2006 & Italy & Caucasian & PCR-RFLP & $\mathrm{HB}$ & $84 / 106$ & CC & $1,2,3$ & 7 \\
\hline Onay & 2006 & Canada & Mixed & TaqMan & PB & $398 / 372$ & CC & 1 & 8 \\
\hline $\begin{array}{l}\text { Balasu } \\
\text { bramanian }\end{array}$ & 2006 & UK & Caucasian & TaqMan & $\mathrm{HB}$ & $497 / 498$ & $\mathrm{CC}$ & 1 & 7 \\
\hline Guzowski & 2005 & America & Mixed & DHPLC & $\mathrm{HB}$ & $50 / 25$ & CC & $1,2,3$ & 7 \\
\hline Langsenlehner & 2005 & Australia & Caucasian & TaqMan & PB & $500 / 496$ & $\mathrm{CC}$ & 3 & 8 \\
\hline Abdolrahim & 2005 & Iran & Caucasian & PCR-RFLP & $\mathrm{HB}$ & $275 / 320$ & CC & $1,2,3$ & 8 \\
\hline Smith & 2004 & UK & Caucasian & ARMS-PCR & $\mathrm{HB}$ & $144 / 263$ & CC & 1 & 8 \\
\hline \multicolumn{10}{|c|}{$\begin{array}{l}\text { SOC, source of controls; HB: hospital-based; PB, population-based;CC, case-control; PCR, polymerase chain reaction; RFLP, } \\
\text { restriction fragment length polymorphism; DHPLC, denaturing highperformance liquid chromatography; EPIC, European } \\
\text { Prospective Investigation of Cancer (a prospective study of diet and cancer being carried out in nine European countries); } \\
\text { ASPCR, allele-specific PCR; SNP, single-nucleotide polymorphisms; SNP No.1, - } 1082 \text { A > G (rs1800896); 2: - 819 T >C } \\
\text { (rs1800871); } 3,-592 \text { A >C (rs1800872). NOS, Newcastle-ottawa scale. }\end{array}$} \\
\hline
\end{tabular}


Table 2

IL-10 Polymorphisms Genotype Distribution and Allele Frequency in Cases and Controls

\begin{tabular}{|c|c|c|c|c|c|c|c|c|c|c|c|c|c|}
\hline \multirow{3}{*}{$\begin{array}{l}\text { First author } \\
\\
-1082 A>G \\
\text { rs } 1800896\end{array}$} & \multirow{3}{*}{ Case } & \multirow{3}{*}{ Control } & \multicolumn{5}{|c|}{ Cases } & \multicolumn{5}{|c|}{ Control } & \multirow[t]{3}{*}{ MAF } \\
\hline & & & \multicolumn{3}{|c|}{ Genotypes } & \multicolumn{2}{|c|}{ Alleles } & \multicolumn{3}{|c|}{ Genotypes } & \multicolumn{2}{|c|}{ Alleles } & \\
\hline & & & AA & $A C$ & $\mathrm{CC}$ & $A$ & C & AA & $A C$ & $\mathrm{CC}$ & $A$ & C & \\
\hline Atoum 2016 & 202 & 210 & 157 & 29 & 16 & 343 & 61 & 151 & 42 & 17 & 344 & 76 & 0.181 \\
\hline Alsuhaibani2016 & 80 & 80 & 16 & 47 & 17 & 79 & 81 & 14 & 50 & 16 & 78 & 82 & 0.512 \\
\hline Vinod 2015 & 125 & 160 & 76 & 31 & 18 & 183 & 67 & 67 & 78 & 15 & 212 & 108 & 0.337 \\
\hline Pooja 2012 & 200 & 200 & 132 & 60 & 8 & 324 & 76 & 145 & 50 & 5 & 34 & 60 & 0.638 \\
\hline Kong2010 & 315 & 322 & 285 & 29 & 1 & 599 & 31 & 285 & 35 & 2 & 605 & 39 & 0.061 \\
\hline Gonullu 2007 & 38 & 24 & 13 & 22 & 3 & 48 & 28 & 16 & 7 & 1 & 39 & 9 & 0.187 \\
\hline Guzowski2005 & 50 & 25 & 10 & 28 & 12 & 48 & 52 & 9 & 12 & 4 & 30 & 20 & 0.400 \\
\hline Sabet 2017 & 105 & 50 & 15 & 41 & 49 & 71 & 139 & 27 & 21 & 2 & 75 & 25 & 0.250 \\
\hline Tian 2017 & 312 & 312 & 51 & 132 & 129 & 234 & 390 & 27 & 154 & 131 & 208 & 416 & 0.666 \\
\hline $\begin{array}{l}\text { Abdolrahim-Zadeh } \\
2005\end{array}$ & 275 & 320 & 119 & 116 & 40 & 171 & 373 & 146 & 125 & 49 & 417 & 223 & 0.348 \\
\hline Scola 2006 & 84 & 106 & 28 & 40 & 16 & 96 & 72 & 40 & 45 & 21 & 125 & 87 & 0.410 \\
\hline Onay 2006 & 398 & 372 & 90 & 205 & 103 & 385 & 411 & 107 & 194 & 71 & 408 & 336 & 0.451 \\
\hline Balasu 2006 & 497 & 498 & 121 & 253 & 123 & 499 & 495 & 117 & 260 & 121 & 494 & 502 & 0.504 \\
\hline Maruthi 2017 & 285 & 285 & 80 & 146 & 59 & 262 & 308 & 89 & 159 & 37 & 234 & 336 & 0.589 \\
\hline Al-Ankoshy2019 & 70 & 70 & 36 & 10 & 24 & 97 & 43 & 16 & 17 & 37 & 44 & 96 & 0.690 \\
\hline Smith 2004 & 144 & 263 & 32 & 58 & 39 & 136 & 122 & 46 & 120 & 57 & 250 & 276 & 0.524 \\
\hline Li2014 & 128 & 128 & 96 & 30 & 2 & 222 & 34 & 80 & 44 & 4 & 204 & 52 & 0.203 \\
\hline $\begin{array}{l}-819 T>C \\
(\mathrm{rs} 1800871)\end{array}$ & & & TT & $\mathrm{TC}$ & $\mathrm{CC}$ & $\mathrm{T}$ & C & TT & $\mathrm{TC}$ & $\mathrm{CC}$ & $\mathrm{T}$ & C & \\
\hline Atoum 2016 & 202 & 210 & 88 & 47 & 67 & 223 & 181 & 93 & 41 & 76 & 227 & 193 & 0.459 \\
\hline Wang2014 & 474 & 501 & 90 & 198 & 186 & 378 & 570 & 48 & 219 & 234 & 315 & 687 & 0.685 \\
\hline Pooja 2012 & 200 & 200 & 54 & 92 & 54 & 200 & 200 & 65 & 78 & 57 & 208 & 192 & 0.480 \\
\hline Kong2010 & 315 & 322 & 119 & 135 & 61 & 273 & 257 & 134 & 131 & 57 & 399 & 245 & 0.380 \\
\hline Gonullu 2007 & 38 & 24 & 5 & 17 & 16 & 27 & 49 & 4 & 10 & 10 & 18 & 30 & 0.625 \\
\hline Guzowski2005 & 50 & 25 & 3 & 19 & 28 & 25 & 75 & 1 & 10 & 14 & 12 & 38 & 0.760 \\
\hline Sabet 2017 & 105 & 50 & 16 & 47 & 42 & 79 & 131 & 26 & 22 & 2 & 74 & 26 & 0.260 \\
\hline Tian 2017 & 312 & 312 & 124 & 141 & 47 & 389 & 235 & 144 & 128 & 40 & 416 & 208 & 0.333 \\
\hline $\begin{array}{l}\text { Abdolrahim-Zadeh } \\
2005\end{array}$ & 275 & 320 & 129 & 120 & 26 & 375 & 172 & 166 & 122 & 32 & 454 & 186 & 0.290 \\
\hline He 2012 & 347 & 500 & 177 & 141 & 29 & 495 & 199 & 229 & 223 & 44 & 681 & 311 & 0.313 \\
\hline
\end{tabular}




\begin{tabular}{|c|c|c|c|c|c|c|c|c|c|c|c|c|c|}
\hline \multirow{2}{*}{$\begin{array}{l}\text { First author } \\
\text { Scola } 2006\end{array}$} & \multirow[b]{2}{*}{84} & \multirow[b]{2}{*}{106} & \multicolumn{5}{|c|}{ Cases } & \multicolumn{5}{|c|}{ Control } & \multirow{2}{*}{$\begin{array}{l}\text { MAF } \\
0.721\end{array}$} \\
\hline & & & 5 & 30 & 49 & 40 & 128 & 12 & 35 & 59 & 59 & 177 & \\
\hline Li2014 & 128 & 128 & 105 & 22 & 1 & 232 & 23 & 96 & 28 & 4 & 220 & 36 & 0.203 \\
\hline $\begin{array}{l}-592 C>A \\
(\text { rs1800872) }\end{array}$ & & & $A A$ & $A C$ & $\mathrm{CC}$ & $A$ & C & $\mathrm{AA}$ & $\mathrm{AC}$ & $\mathrm{CC}$ & $A$ & C & \\
\hline $\begin{array}{l}\text { Gallegos- } \\
\text { Arreola2019 }\end{array}$ & 368 & 320 & 42 & 154 & 172 & 238 & 498 & 11 & 100 & 209 & 122 & 518 & 0.190 \\
\hline Zeng2019 & 208 & 215 & 10 & 88 & 110 & 108 & 308 & 22 & 95 & 98 & 139 & 291 & 0.323 \\
\hline Atoum 2016 & 202 & 210 & 76 & 84 & 42 & 236 & 168 & 79 & 91 & 40 & 249 & 171 & 0.593 \\
\hline Pooja 2012 & 200 & 200 & 45 & 67 & 88 & 157 & 243 & 38 & 84 & 78 & 160 & 240 & 0.400 \\
\hline Kong2010 & 315 & 322 & 119 & 135 & 61 & 373 & 257 & 134 & 131 & 57 & 399 & 245 & 0.620 \\
\hline Gonullu 2007 & 38 & 24 & 5 & 17 & 16 & 27 & 49 & 4 & 10 & 10 & 18 & 30 & 0.375 \\
\hline Guzowski2005 & 50 & 25 & 3 & 17 & 30 & 23 & 77 & 3 & 10 & 12 & 16 & 34 & 0.320 \\
\hline Langsenlehner2005 & 500 & 496 & 21 & 210 & 269 & 252 & 748 & 36 & 199 & 261 & 271 & 721 & 0.273 \\
\hline Sabet 2017 & 105 & 50 & 4 & 36 & 65 & 42 & 166 & 31 & 16 & 6 & 78 & 28 & 0.736 \\
\hline Tian 2017 & 312 & 312 & 131 & 130 & 51 & 392 & 232 & 141 & 127 & 44 & 409 & 215 & 0.655 \\
\hline $\begin{array}{l}\text { Abdolrahim-Zadeh } \\
2005\end{array}$ & 275 & 320 & 27 & 100 & 148 & 154 & 396 & 29 & 132 & 159 & 190 & 450 & 0.297 \\
\hline Scola 2006 & 84 & 106 & 5 & 30 & 49 & 40 & 128 & 12 & 35 & 59 & 59 & 153 & 0.278 \\
\hline Pharoah 2007 & 2045 & 218 & 116 & 679 & 1251 & 367 & 3181 & 116 & 764 & 1338 & 996 & 3440 & 0.225 \\
\hline
\end{tabular}

\section{Meta-analysis results}

The association between IL-10 polymorphism (rs1800896, rs1800871, and rs1800872) and BC were shown in Table 3 and Fig. 25. Squares and horizontal lines correspond to study-specific OR and $95 \% \mathrm{Cl}$. The area of a square reflects the weight (inversely proportional to the variance). The diamond represents the sum of OR and $95 \% \mathrm{Cl}$.

Table 3

The minor allele frequencies of the three IL-10 gene polymorphisms locus

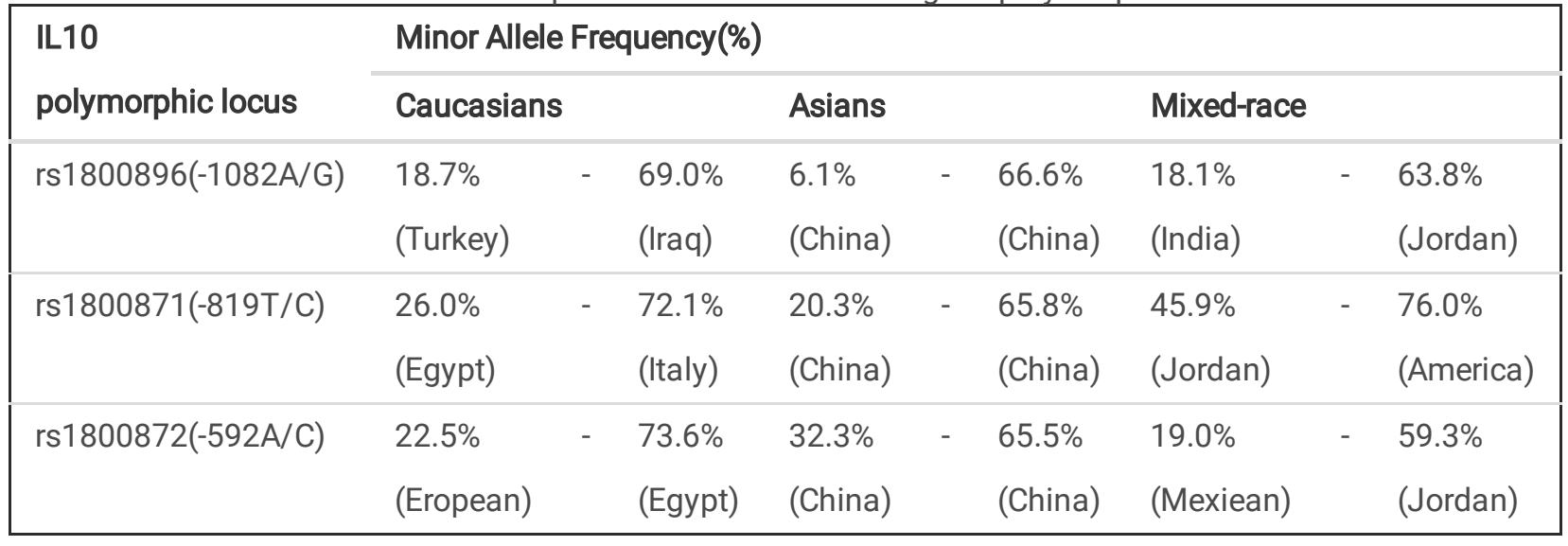


Table 4

Results of the Association of IL-10 Polymorphisms with BC Risk

\begin{tabular}{|c|c|c|c|c|c|c|c|c|}
\hline \multirow[t]{2}{*}{ Subgroup } & \multirow[t]{2}{*}{ Genetic Model } & \multirow[t]{2}{*}{ Type of Model } & \multicolumn{2}{|c|}{ Heterogeneity } & \multicolumn{4}{|c|}{ Odds Ratio } \\
\hline & & & $\mathrm{P}^{2}(\%)$ & $\mathrm{PH}$ & OR & $95 \% \mathrm{Cl}$ & $Z$ test & POR \\
\hline \multicolumn{9}{|l|}{ rs1800896 } \\
\hline \multirow[t]{5}{*}{ Overall } & G vs. A & Random & 82 & $<0.00001$ & 1.06 & $0.87-1.28$ & 0.56 & 0.57 \\
\hline & GG vs. AA & Random & 71 & $<0.00001$ & 1.14 & $0.81-1.59$ & 0.73 & 0.46 \\
\hline & GA vs. AA & Random & 73 & $<0.00001$ & 0.91 & $0.71-1.17$ & 0.74 & 0.46 \\
\hline & $G G+G A$ vs. $A A$ & Random & 79 & $<0.00001$ & 0.99 & $0.76-1.28$ & 0.11 & 0.92 \\
\hline & $G G$ vs. $G A+A A$ & Random & 53 & 0.006 & 1.18 & $0.94-1.48$ & 1.45 & 0.15 \\
\hline \multicolumn{9}{|l|}{ Ethnicity } \\
\hline \multirow[t]{5}{*}{ Asian } & G vs. A & Fixed & 0 & 0.47 & 0.78 & $0.65-0.95$ & 2.49 & 0.01 \\
\hline & GG vs. AA & Fixed & 0 & 0.97 & 0.51 & $0.31-0.84$ & 2.68 & 0.007 \\
\hline & GA vs. AA & Fixed & 24 & 0.27 & 0.6 & $0.44-0.81$ & 3.31 & 0.0009 \\
\hline & $G G+G A$ vs. $A A$ & Fixed & 6 & 0.34 & 0.6 & $0.45-0.81$ & 3.39 & 0.0007 \\
\hline & $G G$ vs. $G A+A A$ & Fixed & 0 & 0.66 & 0.94 & $0.69-1.28$ & 0.39 & 0.7 \\
\hline \multirow[t]{5}{*}{ Caucasian } & G vs. A & Random & 0.0089 & $<0.00001$ & 1.19 & $0.83-1.72$ & 0.94 & 0.35 \\
\hline & GG vs. AA & Random & 0.008 & 0.00001 & 1.21 & $0.67-2.16$ & 0.63 & 0.53 \\
\hline & GA vs. AA & Random & 72 & 0.0008 & 1.09 & $0.73-1.62$ & 0.43 & 0.67 \\
\hline & $G G+G A$ vs. $A A$ & Random & 84 & $<0.00001$ & 1.19 & $0.74-1.91$ & 0.7 & 0.48 \\
\hline & $G G$ vs. $G A+A A$ & Random & 70 & 0.002 & 1.14 & $0.75-1.72$ & 0.61 & 0.55 \\
\hline \multicolumn{9}{|l|}{ rs1800871 } \\
\hline \multirow[t]{5}{*}{ Overall } & C vs. T & Random & 84 & $<0.00001$ & 1.11 & $088-1.39$ & 0.87 & 0.39 \\
\hline & CC vs. TT & Random & 75 & $<0.00001$ & 1.12 & $0.75-1.66$ & 0.55 & 0.58 \\
\hline & CT vs. TT & Random & 67 & 0.0004 & 1.11 & $0.85-1.44$ & 0.77 & 0.44 \\
\hline & $\mathrm{CC}+\mathrm{CT}$ vs. TT & Random & 78 & $<0.00001$ & 1.12 & $0.84-1.50$ & 0.77 & 0.44 \\
\hline & CC vs. CT + TT & Random & 50 & 0.03 & 1 & $0.80-1.25$ & 0.02 & 0.98 \\
\hline \multicolumn{9}{|l|}{ Ethnicity } \\
\hline \multirow[t]{5}{*}{ Asian } & C vs. T & Random & 89 & $<0.00001$ & 0.94 & $0.68-1.31$ & 0.35 & 0.72 \\
\hline & CC vs. TT & Random & 79 & 0.0008 & 0.81 & $0.48-1.40$ & 0.74 & 0.46 \\
\hline & CT vs. TT & Random & 76 & 0.002 & 0.86 & $0.61-1.21$ & 0.88 & 0.38 \\
\hline & $\mathrm{CC}+\mathrm{CT}$ vs. TT & Random & 82 & 0.0001 & 0.84 & $0.58-1.22$ & 0.93 & 0.35 \\
\hline & CC vs. CT + TT & Random & 38 & 0.17 & 0.93 & $0.72-1.20$ & 0.59 & 0.56 \\
\hline \multirow[t]{3}{*}{ Caucasian } & C vs. T & Random & 88 & $<0.0001$ & 1.57 & $0.81-3.05$ & 1.33 & 0.18 \\
\hline & CC vs. TT & Random & 84 & 0.0004 & 1.11 & $0.85-1.44$ & 0.77 & 0.44 \\
\hline & CT vs. TT & Random & 45 & 0.14 & 1.8 & $1.03-3.13$ & 2.07 & 0.04 \\
\hline
\end{tabular}




\begin{tabular}{|c|c|c|c|c|c|c|c|c|}
\hline \multirow[t]{2}{*}{ Subgroup } & \multirow[t]{2}{*}{ Genetic Model } & \multirow[t]{2}{*}{ Type of Model } & \multicolumn{2}{|c|}{ Heterogeneity } & \multicolumn{4}{|c|}{ Odds Ratio } \\
\hline & & & $\left.\right|^{2}(\%)$ & $\mathrm{PH}$ & OR & $95 \% \mathrm{Cl}$ & $Z$ test & POR \\
\hline & $\mathrm{CC}+\mathrm{CT}$ vs. TT & Random & 79 & 0.002 & 2.13 & $0.89-5.13$ & 1.69 & 0.09 \\
\hline & CC vs. CT + TT & Random & 78 & 0.003 & 1.64 & $0.69-3.90$ & 1.12 & 0.26 \\
\hline \multicolumn{9}{|l|}{ rs1800872 } \\
\hline \multirow[t]{5}{*}{ Overall } & A vs. C & Random & 89 & $<0.00001$ & 0.82 & $0.66-1.03$ & 1.7 & 0.09 \\
\hline & AA vs. CC & Random & 83 & $<0.00001$ & 0.71 & $0.46-1.08$ & 1.6 & 0.11 \\
\hline & AC vs. CC & Random & 59 & 0.004 & 0.93 & $0.78-1.11$ & 0.8 & 0.43 \\
\hline & $A A+A C$ vs. $C C$ & Random & 79 & 0.00001 & 0.86 & $0.68-1.08$ & 1.31 & 0.19 \\
\hline & $A A$ vs. $A C+C C$ & Random & 60 & 0.004 & 0.95 & $0.75-1.20$ & 0.44 & 0.66 \\
\hline \multicolumn{9}{|l|}{ Ethnicity } \\
\hline \multirow[t]{5}{*}{ Asian } & A vs. C & Fixed & 0 & 0.54 & 0.85 & $0.74-0.98$ & 2.22 & 0.03 \\
\hline & AA vs. CC & Fixed & 23 & 0.27 & 0.74 & $0.55-0.99$ & 2.04 & 0.04 \\
\hline & $A C$ vs. CC & Fixed & 0 & 0.88 & 0.88 & $0.69-1.13$ & 0.96 & 0.34 \\
\hline & $A A+A C$ vs. $C C$ & Fixed & 0 & 0.81 & 0.82 & $0.65-1.04$ & 1.65 & 0.1 \\
\hline & $A A$ vs. $A C+C C$ & Fixed & 25 & 0.26 & 0.82 & $0.66-1.01$ & 1.84 & 0.07 \\
\hline \multirow[t]{5}{*}{ Caucasian } & A vs. C & Random & 93 & $<0.00001$ & 0.65 & $0.43-0.98$ & 2.07 & 0.04 \\
\hline & AA vs. CC & Random & 89 & $<0.00001$ & 0.43 & $0.19-1.00$ & 1.96 & 0.05 \\
\hline & AC vs. CC & Random & 48 & 0.09 & 0.89 & $0.72-1.11$ & 1.02 & 0.31 \\
\hline & $A A+A C$ vs. $C C$ & Random & 82 & $<0.00001$ & 0.74 & $0.52-1.05$ & 1.69 & 0.09 \\
\hline & $A A$ vs. $A C+C C$ & Random & 36 & 0.18 & 0.87 & $0.62-1,21$ & 0.84 & 0.4 \\
\hline
\end{tabular}

\section{Correlation Between rs1800896 polymorphism and BC}

The 17 studies were conducted on the association between IL-10 rs1800896 polymorphism and BC, with a total sample size of 6,733 cases, 3,308 patients, and 3,425 healthy controls. Overall population heterogeneity test $\mathrm{I}^{2}$ was $82 \%$. The random-effect model results showed that The comparison results of the 5 gene models showed no statistical significance between rs 1800896 polymorphism and $\mathrm{BC}$ (Table 3). Subgroups by race showed that under the four genetic models (allele $\mathrm{G}$ vs. A: $\mathrm{OR}=0.78,95 \% \mathrm{Cl}$ $=0.65-0.95, P=0.01$; homozygous GG vs. AA: $\mathrm{OR}=0.51,95 \% \mathrm{Cl}=0.31-0.84, P=0.007$; heterozygous $\mathrm{GA}$ vs. $\mathrm{AA}: \mathrm{OR}=0.6,95 \% \mathrm{Cl}$ $=0.44-0.81, P=0.0009$; dominant $\mathrm{GG}+\mathrm{GA}$ vs. $\mathrm{AA}: \mathrm{OR}=0.6,95 \% \mathrm{Cl}=0.45-0.81, P=0.0007$ ) (figure. $2 \mathrm{~A}-\mathrm{D}$ ), rs 1800896 polymorphism was significantly associated with $B C$ risk in the Asians. This suggests that race is likely to be the source of heterogeneity and the 4 genetic models of rs 1800896 polymorphism are significantly associated with the BC risk in Asians.

\section{Correlation between rs1800871 polymorphism and BC}

The 12 studies assessed the strength of the association between IL-10 rs 1800871 polymorphism and BC in a total of 2,530 patients and 2,698 controls. There was no association between BC risk and rs 1800871 polymorphism in any genetic model of the general population. However, when stratified by race, the rs 1800871 polymorphism was associated with BC risk in Caucasians in the heterozygous model (CT vs. TT: $\mathrm{OR}=1.8,95 \% \mathrm{Cl}=1.03-3.13, P=0.04)$ (Fig. 3). This result indicates that Caucasians with the rs1800871 heterozygous model are more likely to develop BC than individuals with other genotypes.

\section{Correlation between rs 1800872 polymorphism and BC}


The 13 studies (4702 cases and 4818 controls) assessed the strength of the association between IL-10 rs 1800872 polymorphism and BC. As shown in Table 3, the 5 gene model comparison results all showed the association between IL-10 rs1800872 polymorphism and $\mathrm{BC}$ in the general population was not statistically significant. However, after stratification by race, the homozygous model of rs 1800872 polymorphism was associated with $\mathrm{BC}$ risk in Asians (AA vs. CC: $\mathrm{OR}=0.74,95 \% \mathrm{Cl}=0.55-$ $0.99, P=0.04$ )(Fig. 4A). Allele model of rs 1800872 polymorphism was associated with risk of $B C$ in Asians ( $\mathrm{A}$ vs. $\mathrm{C}: \mathrm{OR}=0.85$, $95 \% \mathrm{Cl}=0.74-0.98, P=0.03$ )(figure. $4 \mathrm{~B}$ ) and Caucasians (A vs. $\mathrm{C}$ : $\mathrm{OR}=0.65,95 \% \mathrm{Cl}=0.43-0.98, P=0.04$ )(figure. $4 \mathrm{C}$ ). In other words, if rs 1800872 is an allelic genetic model, the BC risk is much higher in both Asians and Caucasians than in other genotypes.

\section{Publication bias}

Funnel plot, Begg's test, and Egger's test were used to evaluate the publication bias (Stata12.0). As shown in Fig. 6, the funnel plot is essentially symmetrical and the P values of Begg's test and Egger test are all greater than 0.05 . It is indicated that there is almost no obvious publication bias at the three loci.

\section{Discussion}

IL-10, originally known as cytokine synthesis inhibitory factor (CSIF), is a powerful anti-inflammatory cytokine. IL-10 can stimulate the expression of carboxypeptidase B2 (CPB2) in inflammatory BC cells, thus increasing the aggressiveness of the cancer cells [5]. IL-10 is involved in the abnormal proliferation of breast ducts and lobules and stimulates mitotic activity, leading to an increased risk of cancer $[24,39]$. Studies on the mechanism have shown that the production of IL-10 may represent a new escape mechanism for breast cancer cells to escape the destruction of the immune system. IL-10 gene silencing down-regulated the expression of PI3K/AKT and Bcl2 genes, and increased the cleavage expression levels of BBC3, Bax, caspase3, and caspase3[40]. IL-10 can also induce tumor progression by inhibiting a large number of cytokines such as IL-1a, IL-1b, TNF-DNA, IL-6, IL-8, IL-12, and IL-18. This shows that IL-10 plays an important role in promoting cancer immune escape. This may be closely related to the fact that IL-10 gene polymorphism can change its transcriptional activity or protein structure. These variants may affect the biological function of IL-10, leading to immune dysfunction, affect the anti-tumor immune response, and ultimately increase susceptibility to BC $[24,41,42]$.

Studies have shown that the three most common single nucleotide polymorphisms (SNPs) play an important role in regulating IL10 activity. They were located at the transcriptional starting point of rs1800896 (-1082A/G), rs1800871 (-819T/C) and rs1800872 $(-592 \mathrm{~A} / \mathrm{C})$ and encoded high (GCC), medium (ACC) or low (ATA) expression of IL-10 respectively[43-45]. Several other polymorphic loci of IL10 (rs1800890, rs6703630, and rs6693899) are also controversial, but there are few relevant studies at present. In recent years, race has been involved in many studies on the relationship between IL-10 gene polymorphism and the risk of BC. For example, the IL-10 gene rs 1800872 polymorphism was associated with BC susceptibility in this sample from the Mexican population[16]. Also, there was significant association of mutant allele and genotypes of IL-10 rs1800896 with Indian postmenopausal BC[23]. Since the IL-10 gene polymorphism were associated with the risk of BC, we hypothesized that race is the key to the association between IL-10 polymorphism and BC. This meta-analysis conducted the most comprehensive analysis of the relationship between three IL-10 polymorphisms (rs1800896, rs1800871, and rs1800872) and the BC risk of different races. In a subgroup analysis by ethnicity (Asian and Caucasian/mixed race), the three IL-10 polymorphisms (rs1800896, rs 1800871 , and rs1800872) were significantly associated with $\mathrm{BC}$. It showed that rs 1800896 (allele $\mathrm{G}$ vs. $\mathrm{A}: \mathrm{OR}=0.78,95 \% \mathrm{Cl}=0.65-0.95, P=$ 0.01 ; homozygous GG vs. AA: $\mathrm{OR}=0.51,95 \% \mathrm{Cl}=0.31-0.84, P=0.007$; heterozygous $\mathrm{GA}$ vs. $\mathrm{AA}: \mathrm{OR}=0.6,95 \% \mathrm{Cl}=0.44-0.81, P=$ 0.0009 ; dominant $\mathrm{GG}+\mathrm{GA}$ vs. $\mathrm{AA}: \mathrm{OR}=0.6,95 \% \mathrm{Cl}=0.45-0.81, P=0.0007)$ were significantly correlated with $\mathrm{BC}$ risk in Asia. The rs1800871 heterozygote model (CT vs. TT: $\mathrm{OR}=1.8,95 \% \mathrm{Cl}=1.03-3.13, P=0.04$ ) was associated with $\mathrm{BC}$ risk in the Caucasians. The rs 1800872 homozygous model (AA vs $\mathrm{CC}$ : $\mathrm{OR}=0.74,95 \% \mathrm{Cl}=0.55-0.99, P=0.04$ ) was associated with $\mathrm{BC}$ risk in Asians, and the allelic model (A vs. $\mathrm{C}$ : $\mathrm{OR}=0.85,95 \% \mathrm{Cl}=0.74-0.98, P=0.03$ ) was associated with $\mathrm{BC}$ risk in Asians and Caucasians (A vs $\mathrm{C}: \mathrm{OR}=0.65,95 \%=\mathrm{Cl} 0.43-0.98, P=0.04)$. It is suggested that the ethnic subgroup of IL-10 gene polymorphism is the key factor affecting the susceptibility to BC. 
Previously, Dai et al.[46], Abedinzadeh et al[47] and Moghimi et al. [39] have analyzed the correlation between IL10 polymorphism and breast cancer risk, but their analysis is not comprehensive enough. There are few studies included and the ethnic division is not accurate enough. The quality, quantity and new studies included in the meta-analysis will directly affect the credibility and stability of the results. We used a broad search strategy to capture all relevant information. This meta-analysis conducted a more comprehensive analysis of the relationship between three IL-10 polymorphisms (rs1800896, rs1800871, and rs1800872) and BC risk. 23 studies included in the review span decades (publish between 2004 and 2019). It included the researches both missing and published recently, and ruled out the low quality. So our results more accurately estimate the association between IL-10 polymorphism and BC risk than all previous meta-analysis. In this meta-analysis, the latest, most studies and the quality assessment were good were included. There was no significant publication bias, heterogeneity was small in the subgroups, and the sensitivity analysis results did not change. Therefore, the conclusion of the association between the three IL-10 polymorphisms (rs1800896, rs1800871, and rs1800872) and BC in this meta-analysis is reliable and has certain clinical guidance values. The current meta-analysis is the most comprehensive and has sufficient statistical power to assess the association between IL-10 polymorphism and BC risk.

However, it has several limitations that should be acknowledged. First, due to the limited research on the interaction between these three polymorphic sites and their interaction with the environment, it is impossible to estimate the impact of gene-gene and gene-environment interaction on the results of the study. Secondly, we found that heterogeneity existed in the meta-analysis as indicated by the $\mathrm{I}^{2}$ values. Despite the usage of a random-effects model in some studies, the heterogeneity remained. It is predictable because other factors that affect BC should be considered, such as staging and grading of tumors, age, genetic background, environment, and lifestyle. However, due to the lack of some qualified original data, we are unable to calculate the impact of these factors on $\mathrm{BC}$. Besides, the variation range of the minimum allele frequency is large due to the differences in the regional environment, living habits, ethnic customs, and age (especially in China), which may have a certain impact on the research results, but these are inestimable. In general, the meta-analysis was performed on 23 articles that addressed the specific research question. Moreover, the influence and sensitivity analyses confirmed the robustness of the main results.

\section{Conclusion}

In summary, this meta-analysis provides new ideas for clinical, genetic, and epidemiological studies of breast cancer. Our results show that alleles, homozygotes, heterozygotes, and dominant genotypes of IL-10 rs1800896 are significantly associated with the risk of BC in Asians. The homozygous and allelic patterns of rs1800872 also increase the risk of breast cancer in Asians, while the heterozygous patterns of rs1800871 and rs1800872 increase the risk of breast cancer in Caucasians. IL10 polymorphism may be a key regulator of breast cancer susceptibility. Different ethnic groups can predict breast cancer susceptibility by detecting different IL10 polymorphisms locus. Therefore, vulnerable individuals can be identified by genotypic characteristics, and $\mathrm{BC}$ can be prevented through interventions aimed at high-risk groups. However, the etiology of BC is complex, so we strongly recommend further genetic association studies to explore the effects of gene-gene interactions on disease susceptibility. Largescale multicenter studies can be conducted in the future to further verify the effects of gene-gene and gene-environment interactions on IL-10 polymorphism and BC risk in different environments.

\section{Declarations}

\section{Provenance and peer review}

Not commissioned, externally peer-reviewed.

\section{Ethics approval and consent to participate}

Ethical approval is not applicable.

\section{Consent for publication}

Written informed consent for publication was obtained from all participants. 


\section{Competing interests}

The authors declare no relevant conflict of interest.

\section{Funding}

The present study was supported by the University-level Foundation of the University of South China (nk2020205).

\section{Authors' contributions}

Lijun Li participated in the research design. Lijun Li and Jiangang Cao performed data analysis and wrote or contributed to the writing of the manuscript. Lijun Li, Donghua Li, and Wei Xiong interpreted the data and drafted the manuscript. Memgling Cao conceived the idea and supervised the study. All authors read and approved the final manuscript.

\section{Acknowledgements}

No further acknowledgement.

\section{Availability of data and materials}

The material of this article is original research. All data in this manuscript are available and transparent for readers.

\section{References}

1. Anastasiadi Z, Lianos GD, Ignatiadou E, Harissis HV, Mitsis M. Breast cancer in young women: an overview. Updates Surg. 2017;69:313-7.

2. Sancho-Garnier H, Colonna M. [Breast cancer epidemiology]. Presse Med. 2019;48:1076-84.

3. Oblak T, Zadnik V, Krajc M, Lokar K, Zgajnar J. Breast cancer risk based on adapted IBIS prediction model in Slovenian women aged 40-49 years - could it be better? Radiol Oncol 2020.

4. Mubarik S, Wang F, Fawad M, Wang Y, Ahmad I, Yu C. Trends and Projections in Breast Cancer Mortality among four Asian countries (1990-2017): Evidence from five Stochastic Mortality Models. Sci Rep. 2020;10:5480.

5. Mohamed HT, El-Husseiny N, El-Ghonaimy EA, Ibrahim SA, Bazzi ZA, Cavallo-Medved D, Boffa MB, El-Shinawi M, Mohamed MM. IL-10 correlates with the expression of carboxypeptidase B2 and lymphovascular invasion in inflammatory breast cancer: The potential role of tumor infiltrated macrophages. Curr Probl Cancer. 2018;42:215-30.

6. Roh JW, Kim MH, Seo SS, Kim SH, Kim JW, Park NH, Song YS, Park SY, Kang SB, Lee HP. Interleukin-10 promoter polymorphisms and cervical cancer risk in Korean women. Cancer Lett. 2002;184:57-63.

7. Howell WM, Rose-Zerilli MJ. Cytokine gene polymorphisms, cancer susceptibility, and prognosis. J Nutr. 2007;137:194S199S.

8. Chen L, Shi Y, Zhu X, Guo W, Zhang M, Che Y, Tang L, Yang X, You Q, Liu Z. IL10 secreted by cancerassociated macrophages regulates proliferation and invasion in gastric cancer cells via cMet/STAT3 signaling. Oncol Rep. 2019;42:595-604.

9. Zhao Y, Chen S, Shen F, Long D, Yu T, Wu M, Lin X. In vitro neutralization of autocrine IL10 affects Op18/stathmin signaling in nonsmall cell lung cancer cells. Oncol Rep. 2019;41:501-11.

10. Bhattacharjee HK, Bansal VK, Nepal B, Srivastava S, Dinda AK, Misra MC. Is Interleukin 10 (IL10) Expression in Breast Cancer a Marker of Poor Prognosis? Indian J Surg Oncol. 2016;7:320-5.

11. Azher A, Al-Ankoshy M, Hamid A, Dawood Alatbee A. The impact of IL-10 gene polymorphism on progressive Breast cancer. 2019.

12. Zhu Z, Liu JB, Liu X, Qian L. Association of interleukin 10 rs 1800896 polymorphism with susceptibility to breast cancer: a meta-analysis. J Int Med Res. 2020;48:300060520904863. 
13. Li M, Yue C, Zuo X, Jin G, Wang G, Guo H, Wu F, Huang S, Zhao X. The effect of interleukin 10 polymorphisms on breast cancer susceptibility in Han women in Shaanxi Province. PLoS One. 2020;15:e0232174.

14. Gerger A, Renner W, Langsenlehner T, Hofmann G, Knechtel G, Szkandera J, Samonigg H, Krippl P, Langsenlehner U. Association of interleukin-10 gene variation with breast cancer prognosis. Breast Cancer Res Treat. 2010;119:701-5.

15. Setrerrahmane $\mathrm{S}, \mathrm{Xu} \mathrm{H}$. Tumor-related interleukins: old validated targets for new anti-cancer drug development. Mol Cancer. 2017;16:153.

16. Patricia Gallegos-Arreola M, Zuniga Gonzalez GM, Figuera LE, Puebla Perez AM, Delgado Saucedo JI. Association of the IL10 gene rs1800872 (-592 C > A) polymorphism with breast cancer in a Mexican population. J BUON. 2019;24:2369-76.

17. Fanyu Z, Wenhui I, Shan Z, Wei T, xiaofen Z. Qiujin z: Association of interleukin-10 gene polymorphism with susceptibility of breast cancer in Chinese women of Guangxi province. Guo ji mian yi xue za zhi. 2019;042:464-7.

18. Goske M, Ramachander VR, Sureka K, Jahan P. Association of II10 Gene Polymorphisms (Rs 1800896, Rs1800872) in Breast Cancer Patients. International Journal Of Medical Science And Clinical Invention 2017.

19. Atoum MF. ACC interleukin-10 gene promoter haplotype as a breast cancer risk factor predictor among Jordanian females. Onco Targets Ther. 2016;9:3353-7.

20. AlSuhaibani ES, Kizilbash NA, Malik S, Dasti JI, Al Beladi F, El-Morshedi N. Polymorphisms in promoter regions of IL-6 and IL10 genes in breast cancer: a case-control study. Genet Mol Res 2016, 15.

21. Vinod C, Jyothy A, Vijay Kumar M, Raman RR, Nallari P, Venkateshwari A. A Common SNP of IL-10 (-1082A/G) is Associated With Increased Risk of Premenopausal Breast Cancer in South Indian Women. Iran J Cancer Prev. 2015;8:e3434.

22. Wang Z, Liu QL, Sun W, Yang CJ, Tang L, Zhang X, Zhong XM. Genetic polymorphisms in inflammatory response genes and their associations with breast cancer risk. Croat Med J. 2014;55:638-46.

23. Pooja S, Chaudhary P, Nayak LV, Rajender S, Saini KS, Deol D, Kumar S, Bid HK, Konwar R. Polymorphic variations in IL-1 beta, IL-6 and IL-10 genes, their circulating serum levels and breast cancer risk in Indian women. Cytokine. 2012;60:122-8.

24. Kong F, Liu J, Liu Y, Song B, Wang H, Liu W. Association of interleukin-10 gene polymorphisms with breast cancer in a Chinese population. J Exp Clin Cancer Res. 2010;29:72.

25. Gonullu G, Basturk B, Evrensel T, Oral B, Gozkaman A, Manavoglu O. Association of breast cancer and cytokine gene polymorphism in Turkish women. Saudi Med J. 2007;28:1728-33.

26. Guzowski D, Chandrasekaran A, Gawel C, Palma J, Koenig J, Wang XP, Dosik M, Kaplan M, Chu CC, Chavan S, et al. Analysis of single nucleotide polymorphisms in the promoter region of interleukin-10 by denaturing high-performance liquid chromatography. J Biomol Tech. 2005;16:154-66.

27. Langsenlehner U, Krippl P, Renner W, Yazdani-Biuki B, Eder T, Koppel H, Wascher TC, Paulweber B, Samonigg H. Interleukin-10 promoter polymorphism is associated with decreased breast cancer risk. Breast Cancer Res Treat. 2005;90:113-5.

28. Sabet S, El-Sayed SK, Mohamed HT, El-Shinawi M, Mohamed MM. Inflammatory breast cancer: High incidence of GCC haplotypes (-1082A/G, -819T/C, and - 592A/C) in the interleukin-10 gene promoter correlates with over-expression of interleukin-10 in patients' carcinoma tissues. Tumour Biol. 2017;39:1010428317713393.

29. Tian K, Zhang R, Wang X. Association of interleukin-10 polymorphisms and haplotypes with the risk of breast cancer in northern China. Int J Clin Exp Pathol. 2017;10:6989-96.

30. Abdolrahim-Zadeh H, Hakkakian N, Asadollahi R, Gharesifard B, Sarvari J, Kamali E, Talei A. Interleukin10 Promoter Polymorphisms and Breast Cancer Risk in Iranian Women. Iranian J Immunol 2005, 3.

31. He JR, Chen LJ, Su Y, Cen YL, Tang LY, Yu DD, Chen WQ, Wang SM, Song EW, Ren ZF. Joint effects of Epstein-Barr virus and polymorphisms in interleukin-10 and interferon-gamma on breast cancer risk. J Infect Dis. 2012;205:64-71.

32. Scola L, Vaglica M, Crivello A, Palmeri L, Forte GI, Macaluso MC, Giacalone A, Di Noto L, Bongiovanni A, Raimondi C, et al. Cytokine gene polymorphisms and breast cancer susceptibility. Ann NY Acad Sci. 2006;1089:104-9.

33. Onay VU, Briollais L, Knight JA, Shi E, Wang Y, Wells S, Li H, Rajendram I, Andrulis IL, Ozcelik H. SNP-SNP interactions in breast cancer susceptibility. BMC Cancer. 2006;6:114.

34. Balasubramanian SP, Azmy IA, Higham SE, Wilson AG, Cross SS, Cox A, Brown NJ, Reed MW. Interleukin gene polymorphisms and breast cancer: a case control study and systematic literature review. BMC Cancer. 2006;6:188. 
35. Pharoah PD, Tyrer J, Dunning AM, Easton DF, Ponder BA, Investigators S. Association between common variation in 120 candidate genes and breast cancer risk. PLoS Genet. 2007;3:e42.

36. Smith KC, Bateman AC, Fussell HM, Howell WM. Cytokine gene polymorphisms and breast cancer susceptibility and prognosis. Eur J Immunogenet. 2004;31:167-73.

37. Meijiang L. The relationship between interleukin gene polymorphism and females genetic susceptibility to breast cancer in Western Guangxi. Journal of Youjiang Medical University for Nationalities. 2014;36:339-43.

38. Stang A. Critical evaluation of the Newcastle-Ottawa scale for the assessment of the quality of nonrandomized studies in meta-analyses. Eur J Epidemiol. 2010;25:603-5.

39. Moghimi M, Ahrar H, Karimi-Zarchi M, Aghili K, Salari M, Zare-Shehneh M, Neamatzadeh H. Association of IL-10 rs1800871 and rs1800872 Polymorphisms with Breast Cancer Risk: A Systematic Review and Meta-Analysis. Asian Pac J Cancer Prev. 2018;19:3353-9.

40. Alotaibi MR, Hassan ZK, Al-Rejaie SS, Alshammari MA, Almutairi MM, Alhoshani AR, Alanazi WA, Hafez MM, Al-Shabanah OA. Characterization of Apoptosis in a Breast Cancer Cell Line after IL-10 Silencing. Asian Pac J Cancer Prev. 2018;19:77783.

41. Sheikhpour E, Noorbakhsh P, Foroughi E, Farahnak S, Nasiri R, Neamatzadeh H. A Survey on the Role of Interleukin-10 in Breast Cancer: A Narrative. Rep Biochem Mol Biol. 2018;7:30-7.

42. Hydes TJ, Burton R, Inskip H, Bellis MA, Sheron N. A comparison of gender-linked population cancer risks between alcohol and tobacco: how many cigarettes are there in a bottle of wine? BMC Public Health. 2019;19:316.

43. Hofmann SR, Laass MW, Fehrs A, Schuppan D, Zevallos VF, Salminger D, Mabert K, Hedrich CM. IL10 promoter haplotypes may contribute to altered cytokine expression and systemic inflammation in celiac disease. Clin Immunol. 2018;190:15-21.

44. Zupin L, Polesello V, Catamo E, Crovella S, Segat L. Interleukin-10 gene promoter polymorphisms in celiac patients from north-eastern Italy. Hum Immunol. 2014;75:656-61.

45. Westendorp RG, Langermans JA, Huizinga TW, Elouali AH, Verweij CL, Boomsma DI, Vandenbroucke JP. Genetic influence on cytokine production and fatal meningococcal disease. Lancet. 1997;349:170-3.

46. Dai ZJ, Wang XJ, Zhao Y, Ma XB, Kang HF, Min WL, Lin S, Yang PT, Liu XX: Effects of interleukin-10 polymorphisms (rs1800896, rs1800871, and rs1800872) on breast cancer risk: evidence from an updated meta-analysis. Genet Test Mol Biomarkers 2014, 18:439-445.

47. Abedinzadeh M, Neamatzadeh H, Jafari M, Forat-Yazdi M, Nasiri R, Farahnak S, Foroughi E, Zare-Shehneh M: Association of Interleukin-10 -1082A > G (rs1800896) Polymorphism with Predisposition to Breast Cancer. a Meta-Analysis based on 17 Case-Control Studies. Rev Assoc Med Bras (1992) 2018, 64:756-764.

\section{Figures}




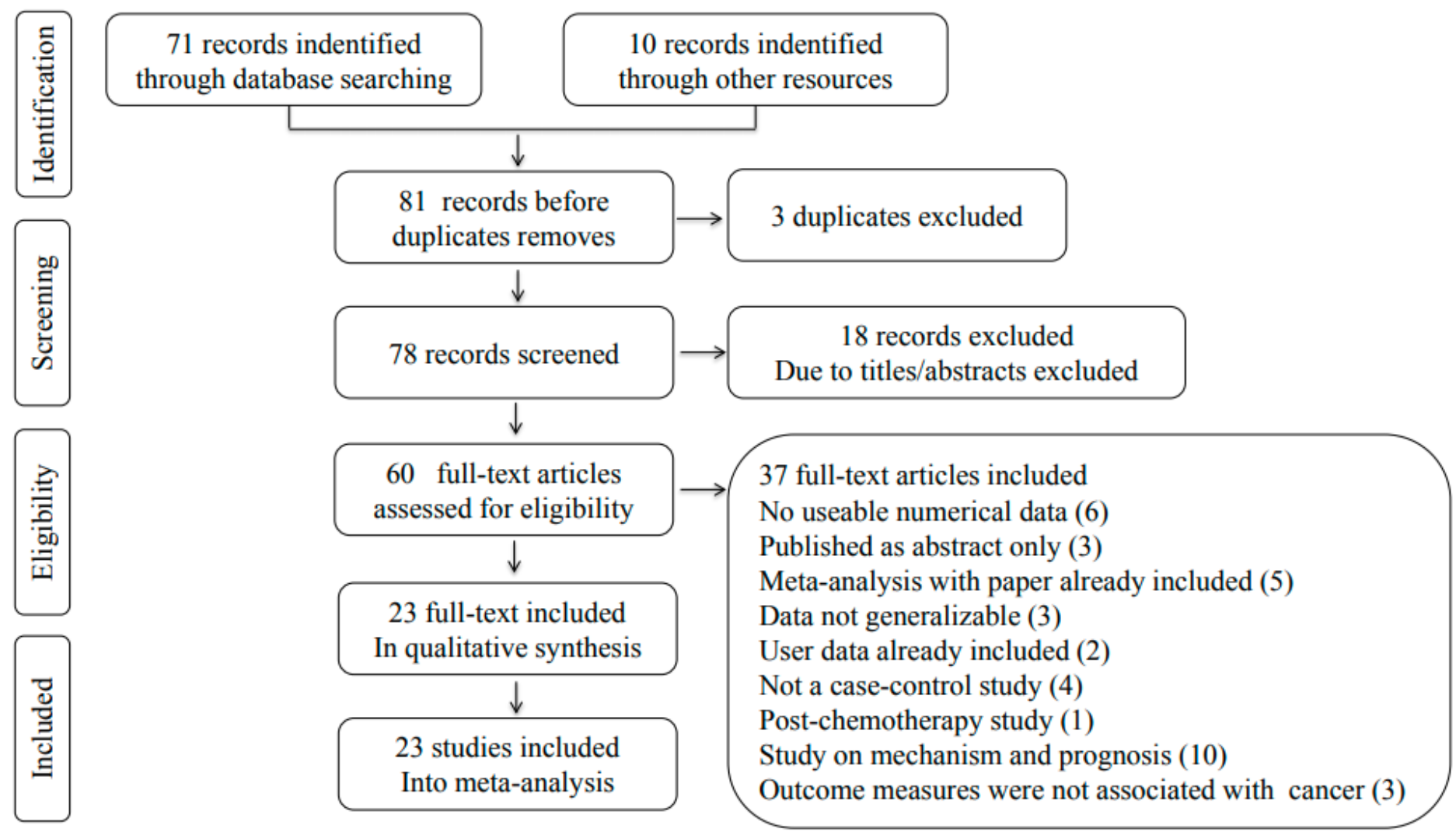

\section{Figure 1}

Flowchart of study selection for the present study.

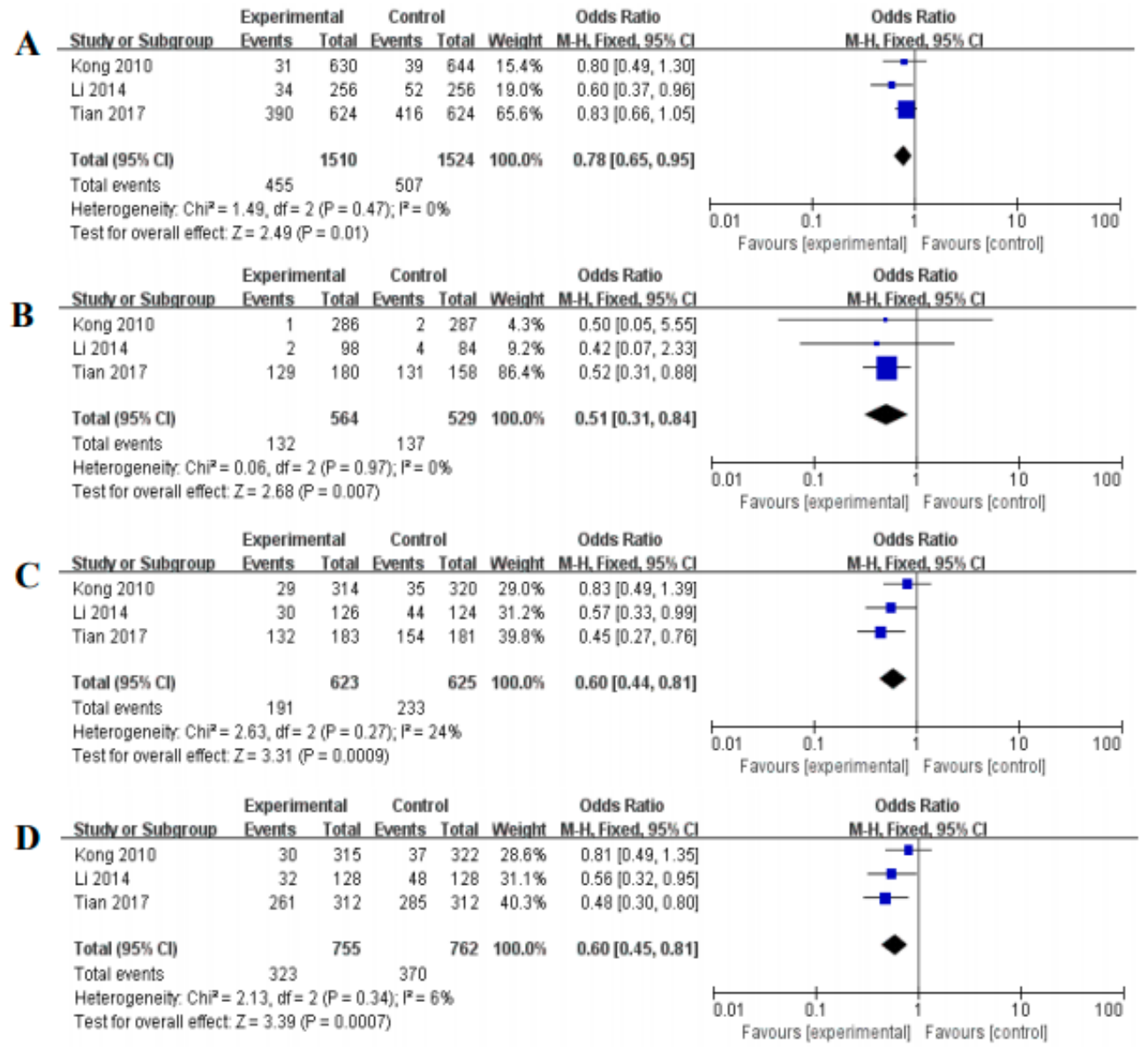


Figure 2

Forest plots of IL-10 rs1800896 polymorphism and BC risk in Asians. $\triangle A$ ) (G vs. A); (B) (GG vs. AA); (C) (GA vs. AA); (D) (GG+GA vs. AA).

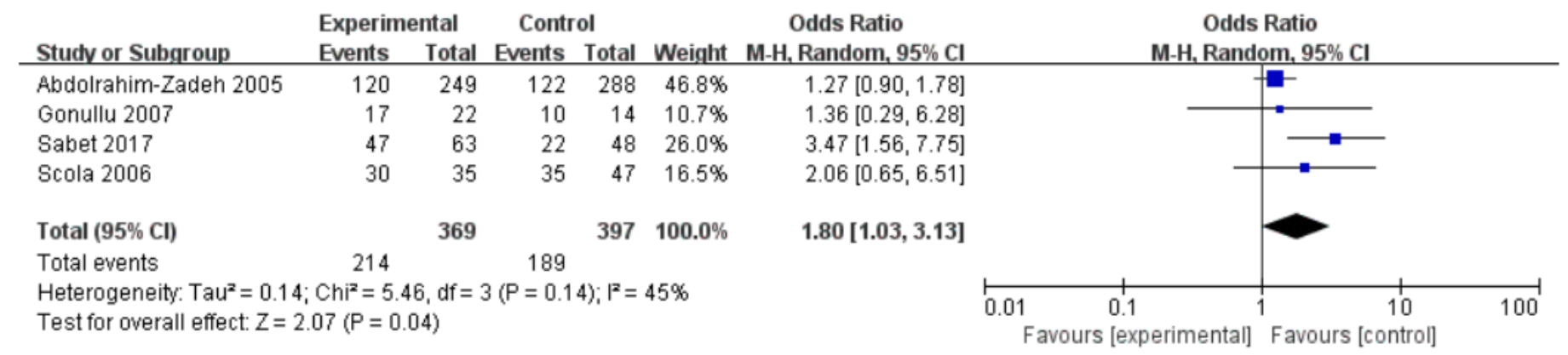

\section{Figure 3}

Forest plots of IL-10 rs1800871 polymorphism and BC risk in the Caucasians $₫$ CT vs. TT区.

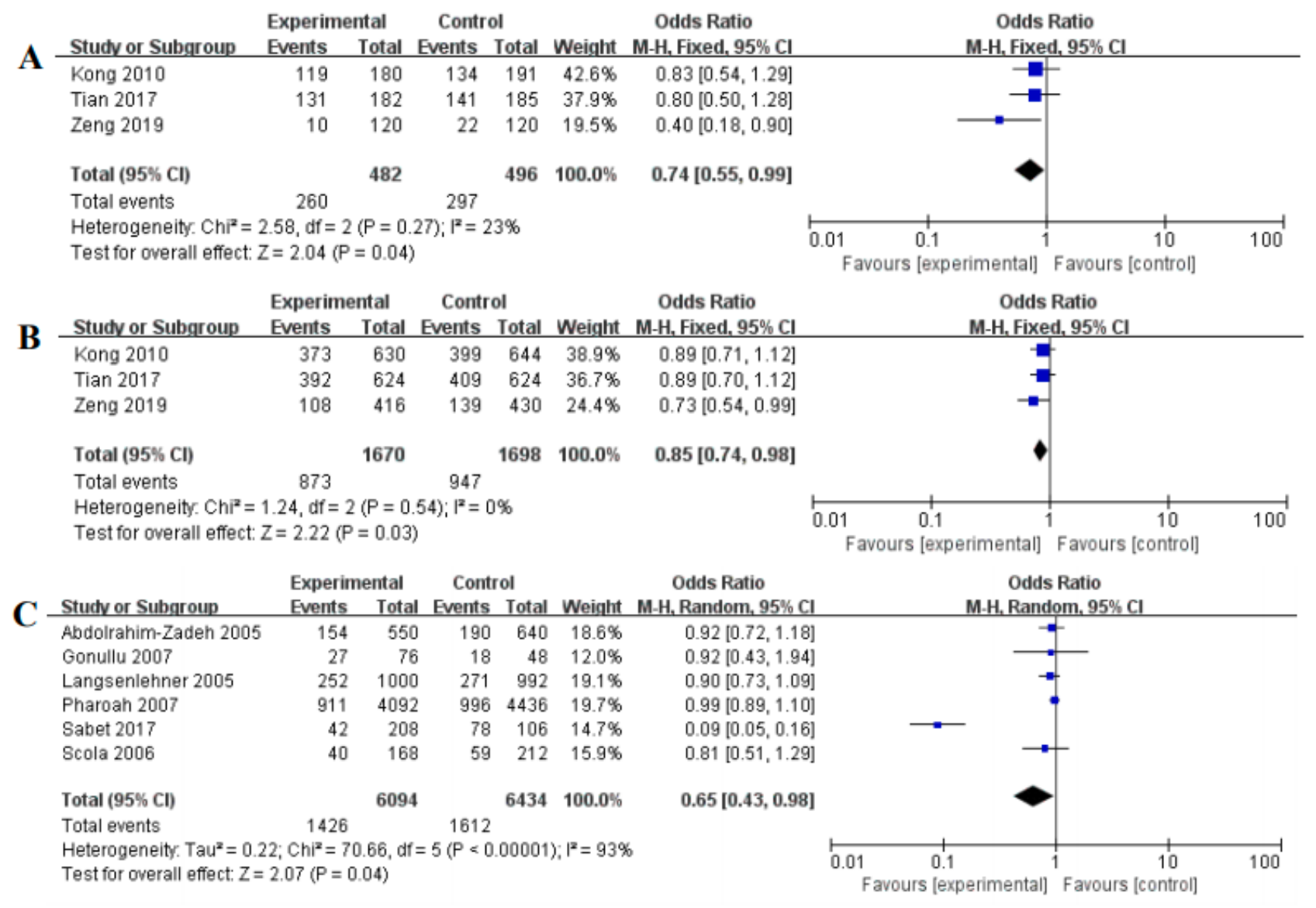

Figure 4

Forest plots of IL-10 rs1800872 polymorphism and breast cancer risk in Asians and Caucasians. (A) IL-10 rs1800872

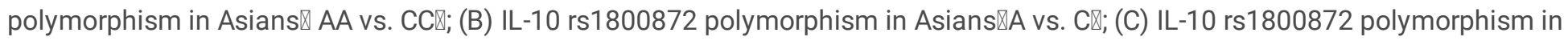

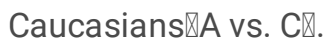


A

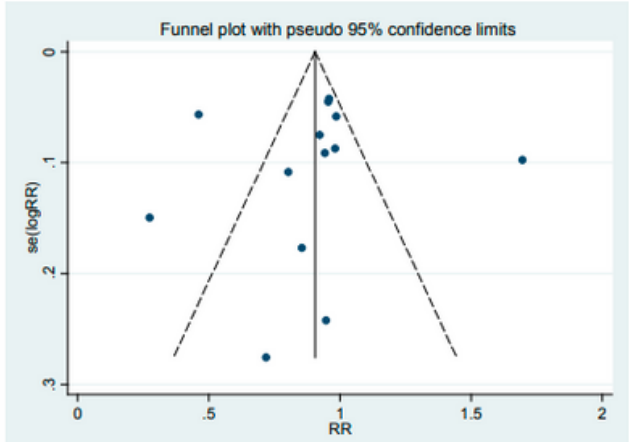

$\mathrm{p}$ value of Begg's test $=0.266$

$\mathrm{p}$ value of Egger's test $=0.972$
B

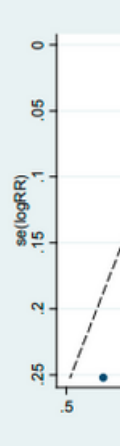

$\mathrm{p}$ value of Begg's test $=0.631$

$\mathrm{p}$ value of Egger's test $=0.90$
C

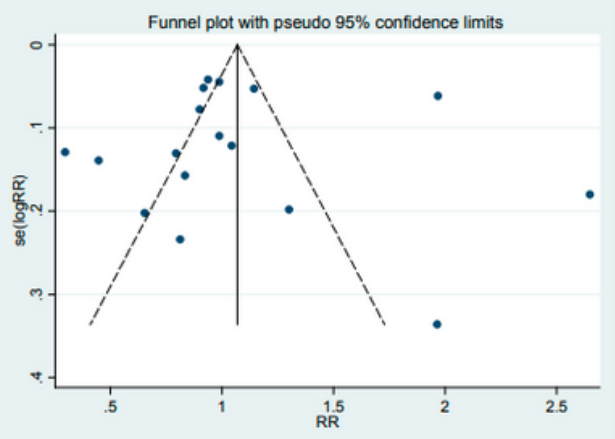

$\mathrm{p}$ value of Begg's test $=0.161$

$p$ value of Egger's test $=0.945$

\section{Figure 5}

Funnel plot of the association between IL-10 gene and breast cancer. (A) rs1800896; (B) rs1800871; (C) rs1800872. 